\title{
DETERMINATION OF THE HOMOLOGY AND THE COHOMOLOGY OF A FEW GROUPS OF ISOMETRIES OF THE HYPERBOLIC PLANE
}

\author{
Nasima Akhter" and Subrata Majumdar \\ Department of Mathematics, \\ Rajshahi University \\ Rajshahi-6205, Bangladesh \\ *Corresponding author: nasima.math.ru@gmail.com \\ Received 07.11.2015 Accepted 10.08.2016
}

\begin{abstract}
In this paper we determine the homology and the cohomology groups of two properly discontinuous groups of isometries of the hyperbolic plane having non-compact orbit spaces and the fundamental group of a graph of groups with a finite vertex groups and no trivial edges by extending Lyndon's partial free resolution for finitely presented groups. For the first two groups, we obtain partial extensions and the corresponding homology. We also compute the corresponding cohomology groups for one of these groups. Finally we obtain homology and cohomology in all dimensions for the last of the above mentioned groups by constructing a full resolution for this group.
\end{abstract}

Keywords: Group presentation, Metacyclic group, Heisenberg group, Free resolution Huebschmann perturbation method, Homology, Cohomology.

\section{Introduction}

In this paper we consider the homology and cohomology of groups of isometries of the hyperbolic plane $H^{2}$.

We recollect that a group $\mathrm{G}$ acts on a space $\mathrm{X}$ properly discontinuously if for any compact subset $\mathrm{C}$ of $\mathrm{X}\{g \in G: g C \cap C \neq \Phi\}$ is finite.

McCullugh and Zimmermann [19] obtained two algebraic characterizations of properly discontinuous groups of isometries of the hyperbolic plane having non compact orbit spaces. One of these characterizations provides a presentation for each such group as is given by the following theorem (see McCullugh and Zimmermann [19]), Theorem 4.1, p.282).

\section{Theorem 1.1}

A group $H$ is a properly discontinuous group of isometries on the hyperbolic plane having noncompact orbit space if and only if $H$ is a (countable) free product of cyclic groups of the form

(i) $\left\langle x_{1}, x_{2}, \ldots,: x_{1}^{2}, x_{2}^{2}, \ldots,\left(x_{1} x_{2}\right)^{n_{1}},\left(x_{2} x_{3}\right)^{n_{2}}, \ldots\right\rangle$ 
where $n_{i}>1$ and the number of generators is finite or infinite,

(ii) $\left\langle\ldots, x_{-1}, x_{0}, x_{1}, x_{2}, \ldots . \ldots, x_{-1}^{2}, x_{0}^{2}, x_{1}^{2}, \ldots\left(x_{-1} x_{0}\right)^{n_{-1}},\left(x_{0} x_{1}\right)^{n_{0}},\left(x_{1} x_{2}\right)^{n_{1}}, \ldots\right\rangle$

and

(iii) $\left\langle x_{1}, \ldots, x_{r}, x_{r+1}: x_{1}^{2}, \ldots, x_{r}^{2},\left(x_{1} x_{2}\right)^{n_{1}}, \ldots,\left(x_{r-1} x_{r}\right)^{n_{r-1}}, x_{r} x_{r+1} x_{1} x_{r+1}^{-1}\right\rangle$.

These presentations were originally obtained by Macbeath and Hoare [8] using geometrical arguments. The main theorem of McCullough and Zimmermann ([19], p.275-276) guarantees that the group $H$ is the fundamental group of a non compact 2-orbifold.

Here we extend Lyndon's 3-term partial resolutions [7] to 6-term partial resolution for the following groups $E$ and $G$, with the use of Fox`s free partial derivatives. The technique of extension has been elaborately described in [18] and this technique has already been used in [1], $[10], \ldots,[17]$.

(a) $E$, which is (iii) of Theorem 1.1,

(b) $G$, which is (i) of Theorem 1.1.

We calculate the homology and cohomology of the group $E$ and the corresponding homology of $G$ up to dimension 4.

The above resolutions can be further extended to full resolutions by similar procedure and the homology and the cohomology calculated.

We also determine the homology and the cohomology of the group $K$ with presentation $\left\langle x_{1}, x_{2}: x_{1}^{2 r}=x_{2}^{2 r}=1, x_{1}^{2}=x_{2}^{2}\right\rangle$. here $r$ is a positive integer.

This group occurs as a subgroup of the extension $\mathrm{X}$ given by $1 \rightarrow F \rightarrow X \rightarrow \mathbf{Z}_{n} \rightarrow 1$, where $F$ is a free group of rank at least 2. $X$ is the fundamental group of a graph of groups with finite vertex groups and no trivial edges. It plays an important role in the proof of Theorem 5.1 of McCullough, Miller and Zimmermann ([19], p. 285).

\section{2. (i). Group $E$ of Hyperbolic Isometries}

Here $E$ is given by $E=\frac{F}{R}$, where $F$ is the free group generated by, say, $x_{1}, x_{2}, \ldots, x_{s}, x_{s+1}$ and $R$ is the normal subgroup of $F$ generated by $r_{1}, r_{2}, \ldots, r_{2 s}$, where

$$
r_{1}=x_{1}^{2}, \ldots, r_{s}=x_{s}^{2}, r_{s+1}=\left(x_{1} x_{2}\right)^{n_{1}}, \ldots, r_{2 s-1}=\left(x_{s-1} x_{s}\right)^{n_{s-1}}, r_{2 s}=x_{s} x_{s+1} x_{1} x_{s+1}^{-1}, s \geq 1 \text { and } n_{i}>1 .
$$

Let $\pi: \mathbf{Z} F \rightarrow \mathbf{Z} E$ be the homomorphism induced by the canonical homomorphism of $F$ onto $E$ with $R$ as the kernel. Let $\pi\left(x_{i}\right)=h_{i}, i=1, \cdots, s+1$.

\section{Theorem 2.1}

The following is a free 6-term $\mathbf{Z} E$-resolution of $\mathbf{Z}$. 


$$
Y_{4} \stackrel{d_{4}}{\rightarrow} Y_{3} \stackrel{d_{3}}{\rightarrow} Y_{2} \stackrel{d_{2}}{\rightarrow} Y_{1} \stackrel{d_{1}}{\rightarrow} Y_{0} \stackrel{d_{0}}{\rightarrow} \mathbf{Z} E \stackrel{\in}{\rightarrow} \mathbf{Z} \rightarrow 0
$$

where

$Y_{0}$ is a right $\mathbf{Z}$ E-module free on $\alpha_{1}, \ldots, \alpha_{s+1}$

$Y_{1},, \quad, \quad, \quad, \quad, \quad, \quad \beta_{1}, \ldots, \beta_{2 s}$

$Y_{2}, \quad, \quad, \quad, \quad, \quad, \quad \delta_{1}, \ldots, \delta_{3 s-1}$

$Y_{3}, \quad, \quad, \quad, \quad, \quad, \quad, \lambda_{1}, \ldots, \lambda_{4 s-2}$

$Y_{4}, \quad, \quad, \quad, \quad, \quad, \quad, \quad \mu_{1}, \ldots, \mu_{5 s-3}$

and $\varepsilon(g)=1 \in \mathbf{Z}$, for $g \in E$,

$d_{0}\left(\alpha_{i}\right)=h_{i}-1, \quad i=1,2, \ldots, s+1$,

$d_{1}\left(\beta_{i}\right)=\alpha_{i}\left(h_{i}+1\right), \quad i=1,2, \ldots, s$,

$\left.\left.d_{1}\left(\beta_{s+i}\right)=\alpha_{i} h_{i+1}\left[\left(h_{i} h_{i+1}\right)^{n_{i}-1}+\ldots+1\right)\right]+\alpha_{i+1}\left[\left(h_{i} h_{i+1}\right)^{n_{i}-1}+\ldots+1\right)\right], \quad i=1,2, \ldots, s-1$,

$d_{1}\left(\beta_{2 s}\right)=\alpha_{1} h_{s+1}^{-1}+\alpha_{s} h_{s}^{-1}+\alpha_{s+1}\left(h_{1} h_{s+1}^{-1}-h_{s+1}^{-1}\right)$

$d_{2}\left(\delta_{i}\right)=\beta_{i}\left(h_{i}-1\right), i=1,2, \ldots, s$.

$\left.d_{2}\left(\delta_{s+i}\right)=-\left(\beta_{i}+\beta_{i+1}\right)\left[\left(h_{i} h_{i+1}\right)^{n_{i}-1}+\ldots+1\right)\right]+\beta_{s+i}\left(h_{i+1}+1\right), \quad i=1,2, \ldots, s-1$,

$d_{2}\left(\delta_{2 s}\right)=-\beta_{1} h_{1}^{-1} h_{s+1}^{-1}-\beta_{s} h_{s}^{-1}+\beta_{2 s}\left(h_{s}+1\right)$

$d_{2}\left(\delta_{2 s+i}\right)=\beta_{s+i}\left(h_{i} h_{i+1}-1\right), i=1,2, \ldots, s-1$.

$d_{3}\left(\lambda_{i}\right)=\delta_{i}\left(h_{i}+1\right), \quad i=1,2, \ldots, s$,

$\left.d_{3}\left(\lambda_{s+i}\right)=\left(\delta_{i}+\delta_{i+1}\right)\left[\left(h_{i} h_{i+1}\right)^{n_{i}-1}+\ldots+1\right)\right]+\delta_{s+i}\left(h_{i+1}-1\right), \quad i=1,2, \ldots, s-1$,

$d_{3}\left(\lambda_{2 s}\right)=\delta_{1} h_{1}^{-1} h_{s+1}+\delta_{s} h_{s}^{-1}+\delta_{2 s}\left(h_{s}^{-1}\right)$

$\left.d_{3}\left(\lambda_{2 s+i}\right)=\delta_{2 s+i}\left[\left(h_{i} h_{i+1}\right)^{n_{i}-1}+\ldots+1\right)\right], \quad i=1,2, \ldots, s-1$,

$\left.d_{3}\left(\lambda_{3 s-1+i}\right)=\delta_{s+i}\left(h_{i} h_{i+1}-1\right)-\delta_{2 s+i}\left[\left(h_{i} h_{i+1}\right)^{n_{i}-2}+\ldots+1\right)\right]\left(h_{i+1}+1\right), \quad i=1,2, \ldots, s-1$,

$d_{4}\left(\mu_{i}\right)=\lambda_{i}\left(h_{i}-1\right), \quad i=1,2, \ldots, s$,

$\left.d_{4}\left(\mu_{s+i}\right)=-\left(\lambda_{i}+\lambda_{i+1}\right)\left[\left(h_{i} h_{i+1}\right)^{n_{i}-1}+\ldots+1\right)\right]+\lambda_{s+i}\left(h_{i+1}+1\right), \quad i=1,2, \ldots, s-1$,

$d_{4}\left(\mu_{2 s}\right)=-\lambda_{1} h_{1}^{-1} h_{s+1}-\lambda_{s} h_{s}^{-1}+\lambda_{2 s}\left(h_{s}+1\right)$

$d_{4}\left(\mu_{2 s+i}\right)=\lambda_{2 s+i}\left(h_{i} h_{i+1}-1\right), i=1,2, \ldots, s-1$.

$\left.d_{4}\left(\mu_{3 s-1+i}\right)=\lambda_{3 s-1+i}\left[\left(h_{i} h_{i+1}\right)^{n_{i}-1}+\ldots+1\right]+\lambda_{2 s+i}\left[\left(h_{i} h_{i+1}\right)^{n_{i}-2}+\ldots+1\right)\right]\left(h_{i+1}+1\right), \quad i=1,2, \ldots, s-1$,

$\left.d_{4}\left(\mu_{4 s-2+i}\right)=\lambda_{s+i}\left(h_{i} h_{i+1}-1\right)-\lambda_{3 s-1+i}\left[\left(h_{i} h_{i+1}\right)^{n_{i}-2}+\ldots+1\right)\right]\left(h_{i+1}-1\right), \quad i=1,2, \ldots, s-1$, 
Let $A$ be a left $Z E$-module, then the homology groups $H_{n}(E, A)$ are given by the homology of the complex:

$$
A^{5 s-3} \stackrel{\bar{d}_{4}}{\rightarrow} A^{4 s-2} \stackrel{\bar{d}_{3}}{\rightarrow} A^{3 s-1} \stackrel{\bar{d}_{2}}{\rightarrow} A^{2 s} \stackrel{\bar{d}_{1}}{\rightarrow} A^{s+1} \stackrel{\bar{d}_{0}}{\rightarrow} A \rightarrow 0
$$

where $A^{k}$ stands for the direct sum of $k$ isomorphic copies of $A$ and the homomorphisms $\bar{d}_{0}, \bar{d}_{1}, \bar{d}_{2}, \bar{d}_{3}, \bar{d}_{4}$ are induced by $d_{0}, d_{1}, d_{2}, d_{3}, d_{4}$ respectively and are given by

$$
\begin{aligned}
& \bar{d}_{0}\left(a_{1}, \ldots, a_{s+1}\right)=\left(h_{1}-1\right) a_{1}+\ldots+\left(h_{s+1}-1\right) a_{s+1}, \\
& \bar{d}_{1}\left(a_{1}, \ldots, a_{2 s}\right)=\left(\left(h_{1}+1\right) a_{1}+h_{2}\left[\left(h_{1} h_{2}\right)^{n_{1}-1}+\ldots+1\right] a_{s+1}+\ldots+h_{s+1}^{-1} a_{2 s},\left(h_{2}+1\right) a_{2}+\right. \\
& {\left[\left(h_{1} h_{2}\right)^{n_{1}-1}+\ldots+1\right] a_{s+1}+h_{3}\left[\left(h_{2} h_{3}\right)^{n_{2}-1}+\ldots+1\right] a_{s+2}, \ldots,\left(h_{s}+1\right) a_{s}+} \\
& \left.\left[\left(h_{s-1} h_{s}\right)^{n_{s-1}-1}+\ldots+1\right] a_{2 s-1}+\ldots+h_{s}^{-1} a_{2 s},\left(h_{1} h_{s+1}^{-1}-h_{s+1}^{-1}\right) a_{2 s}\right), \\
& \bar{d}_{2}\left(a_{1}, \ldots, a_{3 s-1}\right)=\left(\left(h_{1}-1\right) a_{1}-\left[\left(h_{1} h_{2}\right)^{n_{1}-1}+\ldots+1\right] a_{s+1}-h_{1}^{-1} h_{s+1}^{-1} a_{2 s}, \quad\left(h_{2}-1\right) a_{2}-\right. \\
& {\left[\left(h_{1} h_{2}\right)^{n_{1}-1}+\ldots+1\right] a_{s+1}-\left[\left(h_{2} h_{3}\right)^{n_{2}-1}+\ldots+1\right] a_{s+2}, \ldots, \quad\left(h_{2}+1\right) a_{s+1}+} \\
& \left(h_{1} h_{2}-1\right) a_{2 s+1}, \ldots,\left(h_{s}+1\right) a_{2 s-1}+\left(h_{s-1} h_{s}^{-1}\right) a_{3 s-1},\left(h_{s}+1\right) a_{2 s}, \\
& \bar{d}_{3}\left(a_{1}, \ldots, a_{4 s-2}\right)=\left(\left(h_{1}+1\right) a_{1}+\left[\left(h_{1} h_{2}\right)^{n_{1}-1}+\ldots+1\right] a_{s+1}+h_{1}^{-1} h_{s+1}^{-1} a_{2 s}, \quad\left(h_{2}+1\right) a_{2}+\right. \\
& {\left[\left(h_{1} h_{2}\right)^{n_{1}-1}+\ldots+1\right] a_{s+1}+\left[\left(h_{2} h_{3}\right)^{n_{2}-1}+\ldots+1\right] a_{s+2}, \ldots, \quad\left(h_{2}-1\right) a_{s+1}+} \\
& \left(h_{1} h_{2}-1\right) \alpha_{3 s-1+1},\left(h_{s}-1\right) a_{2 s},\left[\left(h_{1} h_{2}\right)^{n_{1}-1}+\ldots+1\right] a_{2 s+1} \\
& -\left[\left(h_{1} h_{2}\right)^{n_{1}-2}+\ldots+1\right]\left(h_{2}+1\right) a_{3 s-1+1}, \ldots,\left[\left(h_{s-1} h_{s}\right)^{n_{s-1}-1}+\ldots+1\right] a_{3 s-1}- \\
& \left.\left[\left(h_{s-1} h_{s}\right)^{n_{s-1}-2}+\ldots+1\right]\left(h_{s}+1\right) a_{4 s-2}\right) \text {, } \\
& \bar{d}_{4}\left(a_{1}, \ldots, a_{4 s-2}\right)=\left(\left(h_{1}-1\right) a_{1}-\left[\left(h_{1} h_{2}\right)^{n_{1}-1}+\ldots+1\right] a_{s+1}-h_{s+1}^{-1} a_{2 s}, \quad\left(h_{2}+1\right) a_{2}-\right. \\
& {\left[\left(h_{1} h_{2}\right)^{n_{1}-1}+\ldots+1\right] a_{s+1}-\left[\left(h_{2} h_{3}\right)^{n_{2}-1}+\ldots+1\right] a_{s+2}, \ldots, \quad\left(h_{2}+1\right) a_{s+1}+\left(h_{1} h_{2}-1\right) a_{4 s-2+1}, \ldots,} \\
& \left(h_{s}+1\right) a_{2 s},\left(h_{1} h_{2}-1\right) a_{2 s+1}+\left[\left(h_{1} h_{2}\right)^{n_{1}-2}+\ldots+1\right]\left(h_{2}+1\right) a_{3 s-1+1}, \ldots,\left[\left(h_{1} h_{2}\right)^{n_{1}-1}+\ldots+1\right] a_{3 s-1+1}- \\
& {\left[\left(h_{1} h_{2}\right)^{n_{1}-2}+\ldots+1\right]\left(h_{2}-1\right) a_{4 s-2+1}, \ldots,\left[\left(h_{s-1} h_{s}\right)^{n_{s-1}-1}+\ldots+1\right] a_{4 s-2}-} \\
& \left.\left[\left(h_{s-1} h_{s}\right)^{n_{s-1}-2}+\ldots+1\right]\left(h_{s}-1\right) a_{5 s-3}\right) \text {, }
\end{aligned}
$$

Hence the integral homology groups of $E$ are

$H_{0}(E, \mathbf{Z}) \cong \mathbf{Z}$.

$$
\begin{aligned}
H_{1}(E, \mathbf{Z}) & \cong \frac{\left\{\left(a_{1}, \ldots, a_{s+1}\right): a_{i} \in \mathbf{Z}\right\}}{\left\{\left(2 a_{1}+n_{1} a_{s+1}+a_{2 s}, 2 a_{2}+n_{1} a_{s+1}+n_{2} a_{s+2}, \ldots, 2 a_{s}+n_{s-1} a_{2 s-1}+a_{2 s}, 0\right)\right\}} \\
& \cong \frac{\left\langle x_{1}\right\rangle \oplus\left\langle x_{2}\right\rangle \oplus \ldots \oplus\left\langle x_{s+1}\right\rangle}{\left\langle 2 x_{1}, \ldots, 2 x_{s}, n_{1}\left(x_{1}+x_{2}\right), n_{2}\left(x_{1}+x_{2}\right), \ldots, n_{s-1} x_{1}, x_{1}+x_{s}\right\rangle},
\end{aligned}
$$


$\cong\left\langle x_{1}, x_{2}, \ldots, x_{s+1} \mid 2 x_{1}=0, \ldots, 2 x_{s}=0, n_{1}\left(x_{1}+x_{2}\right)=0, n_{2}\left(x_{2}+x_{3}\right)=0, \ldots, n_{s-1} x_{s}=0, x_{1}+x_{s}=0\right\rangle$

$\cong \mathbf{Z}_{\infty}\left(x_{s+1}\right) \oplus \mathbf{Z}_{2}\left(x_{i_{1}}\right) \oplus \ldots \mathbf{Z}_{2}\left(x_{i_{r}}\right), \mathrm{r}$ is the number of even $\mathrm{n}_{\mathrm{i}}{ }^{\prime} \mathrm{s}, 2 \leq i \leq s-1$.

$H_{2}(E, \mathbf{Z})=$

$\frac{\left\{\left(a_{1}, \ldots, a_{2 s}\right): 2 a_{1}+n_{1} a_{s+1}+a_{2 s}=2 a_{2}+n_{1} a_{s+1}+n_{2} a_{s+2}=0, \ldots, 0=2 a_{s}+n_{s-1} a_{2 s-1}+a_{2 s} ; a_{i} \in \mathbf{Z}\right\}}{\left\{\left(-n_{1} a_{s+1}-a_{2 s},-n_{1} a_{s+1}-n_{1} a_{s+1}-n_{2} a_{s+2}, \ldots,-n_{s-11} a_{2 s-1}-a_{2 s}, 2 a_{s+1}, \ldots, 2 a_{2 s-1}, 2 a_{2 s}\right) \mid a_{i} \in \mathbf{Z}\right\}} \cong 0$

$H_{3}(E, \mathbf{Z})=\frac{\left\{\left(a_{1}, \ldots, a_{2 s}, a_{2 s+1}, \ldots a_{3 s-1}\right):-n_{1} a_{s+1}=a_{2 s}, n_{1} a_{s+1}=-n_{2} a_{s+2}, \ldots, n_{s-1} a_{2 s-1}=-a_{2 s},\right.}{\left.2 a_{s+1}=0, \ldots, 2 a_{2 s+1}=0, a_{2 s}=0 ; a_{i} \in \mathbf{Z}\right\}}$

If we write $x_{i}$ for $(0, \ldots, 0,1,0, \ldots, 0)$, where 1 is in the $\mathrm{i}$-th position, then

$$
\begin{aligned}
H_{3}(E, \mathbf{Z}) \cong & \left\langle x_{1}, x_{2}, \ldots, x_{s}, x_{2 s+1}, \ldots, x_{3 s-1}: 2 x_{1}=0, \ldots, 2 x_{s}=0, n_{1}\left(x_{1}+x_{2}\right)=0, n_{s-1} x_{s}=0, x_{1}+x_{s}=0,\right. \\
& \left.n_{1} x_{2 s+1}=0,-2\left(n_{1}-1\right) x_{2 s+1}=0, \ldots, 2\left(n_{s-1}-1\right) x_{3 s-1}=0\right\rangle \\
\cong & \mathbf{Z}_{2}\left(x_{i_{1}}\right) \oplus \ldots \oplus \mathbf{Z}_{2}\left(x_{i_{r}}\right) \oplus \mathbf{Z}_{2}\left(x_{j_{1}}\right) \oplus \ldots \oplus \mathbf{Z}_{2}\left(x_{j_{r}}\right), \text { where } r \text { is the number of even } \mathrm{n}_{\mathrm{i}}, \\
& 2 \leq i_{1}, \ldots, i_{r} \leq s \text { and } 2 s+1 \leq j_{1}, \ldots, j_{r} \leq 3 s-1 . \\
H_{4}(E, \mathbf{Z}) \cong & 0 .
\end{aligned}
$$

Let $A$ be a right $Z E$-module, then the cohomology groups $H^{n}(E, A)$ are given by the homology of the complex:

$A^{5 s-3} \stackrel{d_{4}^{*}}{\leftarrow} A^{4 s-2} \stackrel{d_{3}^{*}}{\leftarrow} A^{3 s-1} \stackrel{d_{2}^{*}}{\leftarrow} A^{2 s} \stackrel{d_{1}^{*}}{\leftarrow} A^{s+1} \stackrel{d_{0}^{*}}{\leftarrow} A \leftarrow 0$

where the homomorphisms $d_{0}^{*}, d_{1}^{*}, d_{2}^{*}, d_{3}^{*}, d_{4}^{*}$ are induced by $d_{0}, d_{1}, d_{2}, d_{3}, d_{4}$ respectively and are given by

$$
\begin{aligned}
& d_{0}^{*}(a)=\left(a\left(h_{1}-1\right), \ldots, a\left(h_{s+1}-1\right)\right), \\
& d_{1}^{*}\left(a_{1}, \ldots, a_{s+1}\right)=\left(a_{1}\left(h_{1}+1\right), \ldots, a_{s}\left(h_{s}+1\right), a_{1} h_{2}\left[\left(h_{1} h_{2}\right)^{n_{1}-1}+\ldots+1\right]+a_{2}\left[\left(h_{1} h_{2}\right)^{n_{1}-1}+\ldots+1\right], \ldots,\right. \\
& a_{s-1} h_{s}\left[\left(h_{s-1} h_{s}\right)^{n_{s-1}-1}+\ldots+1\right]+a_{s}\left[\left(h_{s-1} h_{s}\right)^{n_{s-1}-1}+\ldots+1\right], a_{1} h_{s+1}^{-1}+a_{s} h_{s}^{-1}+ \\
& \left.a_{s+1}\left(h_{1} h_{s+1}^{-1}-h_{s+1}^{-1}\right)\right), \\
& d_{2}^{*}\left(a_{1}, \ldots, a_{2 s}\right)=\left(a_{1}\left(h_{1}-1\right), \ldots, a_{s}\left(h_{s}-1\right),-\left(a_{1}+a_{2}\right)\left[\left(h_{1} h_{2}\right)^{n_{1}-1}+\ldots+1\right]+a_{s+1}\left(h_{2}+1\right), \ldots,-\right. \\
& \left(a_{s-1}+a_{s}\right)\left[\left(h_{s-1} h_{s}\right)^{n_{s-1}-1}+\ldots+1\right]+a_{2 s-1}\left(h_{s}+1\right),-a_{1} h_{1}^{-1} h_{s+1}^{-1}-a_{s} h_{s}^{-1}+a_{2 s}\left(h_{s}+1\right), \\
& \left.a_{s+1}\left(h_{1} h_{2}-1\right), \ldots, a_{2 s-1}\left(h_{s-1} h_{s}-1\right)\right), \\
& d_{3}^{*}\left(a_{1}, \ldots, a_{3 s-1}\right)=\left(a_{1}\left(h_{1}+1\right), \ldots, a_{s}\left(h_{s}+1\right),\left(a_{1}+a_{2}\right)\left[\left(h_{1} h_{2}\right)^{n_{1}-1}+\ldots+1\right]+a_{s+1}\left(h_{2}-1\right), \ldots,\right.
\end{aligned}
$$




$$
\begin{gathered}
\left(a_{s-1}+a_{s}\right)\left[\left(h_{s-1} h_{s}\right)^{n_{s-1}-1}+\ldots+1\right]+a_{2 s-1}\left(h_{s}-1\right), a_{1} h_{1}^{-1} h_{s+1}^{-1}+a_{s} h_{s}^{-1}+a_{2 s}\left(h_{s}-1\right), \\
a_{2 s+1}\left[\left(h_{1} h_{2}\right)^{n_{1}-1}+\ldots+1\right], \ldots, a_{s+1}\left(h_{1} h_{2}-1\right)-a_{2 s+1}\left[\left(h_{1} h_{2}\right)^{n_{1}-2}+\ldots+1\right]\left(h_{2}+1\right), \\
\left.a_{2 s-1}\left(h_{s-1} h_{s}-1\right)-a_{3 s-1}\left[\left(h_{s-1} h_{s}\right)^{n_{s-1}-2}+\ldots+1\right]\left(h_{s}+1\right)\right), \\
d_{4}^{*}\left(a_{1}, \ldots, a_{4 s-2}\right)=\left(a_{1}\left(h_{1}-1\right), \ldots, a_{s}\left(h_{s}-1\right),-\left(a_{1}+a_{2}\right)\left[\left(h_{1} h_{2}\right)^{n_{1}-1}+\ldots+1\right]+a_{s+1}\left(h_{2}+1\right), \ldots,-\right. \\
\left(a_{s-1}+a_{s}\right)\left[\left(h_{s-1} h_{s}\right)^{n_{s-1}-1}+\ldots+1\right]+a_{2 s-1}\left(h_{s}+1\right),-a_{1} h_{s+1}^{-1}-a_{s} h_{s}^{-1}+a_{2 s}\left(h_{s}+1\right), \\
a_{2 s+1}\left(h_{1} h_{2}-1\right), \ldots, a_{3 s}\left[\left(h_{1} h_{2}\right)^{n_{1}-1}+\ldots+1\right]+a_{2 s+1}\left[\left(h_{1} h_{2}\right)^{n_{1}-2}+\ldots+1\right]\left(h_{2}+1\right), \ldots, \\
a_{4 s-2}\left[\left(h_{s-1} h_{s}\right)^{n_{s-1}-1}+\ldots+1\right]+a_{3 s-1}\left[\left(h_{s-1} h_{s}\right)^{n_{s-1}-2}+\ldots+1\right]\left(h_{s}+1\right), a_{s+1}\left(h_{1} h_{2}-1\right)- \\
\left.a_{3 s}\left[\left(h_{1} h_{2}\right)^{n_{1}-2}+\ldots+1\right]\left(h_{2}-1\right), \ldots, a_{2 s-1}\left(h_{s-1} h_{s}-1\right)-a_{4 s-1}\left[\left(h_{s-1} h_{s}\right)^{n_{s-1}-1}+\ldots+1\right]\left(h_{s}-1\right)\right),
\end{gathered}
$$

Therefore, the integral cohomology groups of $E$ are

$H^{0}(E, \mathbf{Z}) \cong \mathbf{Z}$.

$H^{1}(E, \mathbf{Z}) \cong \mathbf{Z}$.

$H^{2}(E, \mathbf{Z}) \cong 0$

$H^{3}(E, \mathbf{Z})$

$$
\begin{aligned}
& \cong \frac{\left\{\left(a_{1}, \ldots, a_{3 s-1}\right) \mid \begin{array}{l}
2 a_{1}=0 \ldots=2 a_{s},\left(a_{1}+a_{2}\right) n_{1}=0, \ldots, a_{1}+a_{s}=0, n_{1} a_{2 s+1}=0=n_{s-1} a_{3 s-1}, \\
\left.-2\left(n_{1}-1\right) a_{2 s+1}-2\left(n_{s-1}-1\right) a_{3 s-1}=0 ; a_{i} \in \mathbf{Z}\right\}
\end{array}\right.}{\left\{\left(0, \ldots, 0,-\left(a_{1}+a_{2}\right) n_{1}+2 a_{s+1}, \ldots,-\left(a_{s-1}+a_{s}\right) n_{s-1}+2 a_{2 s-1}-a_{1}-a_{s}+2 a_{2 s}, 0, \ldots, 0\right\}\right.} \\
& \cong \frac{\left\{\left(0, \ldots, 0, a_{s+1}, a_{s+2}, \ldots, a_{2 s}, 0, \ldots, 0\right) \mid a_{i} \in \mathbf{Z}\right\}}{\left\{\left(0, \ldots, 0,2 a_{s+1}-\left(a_{1}+a_{2}\right) n_{1}, \ldots, 2 a_{2 s-1}-\left(a_{s-1}+a_{s}\right) n_{s-1},-a_{1}-a_{s}+2 a_{2 s}, 0, \ldots, 0\right\}\right.} \\
& \cong \frac{\left\{\left(a_{s+1}, a_{s+2}, \ldots, a_{2 s}\right) \mid a_{i} \in \mathbf{Z}\right\}}{\left\{\left(2 a_{s+1}-\left(a_{1}+a_{2}\right) n_{1}, \ldots, 2 a_{2 s-1}-\left(a_{s-1}+a_{s}\right) n_{s-1},-a_{1}-a_{s}+2 a_{2 s}\right\}\right.},
\end{aligned}
$$

if we write $x_{i}=(0, \ldots, 0,1,0, \ldots, 0)$, where 1 is in the $i$-th position, then

$$
\begin{aligned}
& \cong \frac{\left\langle x_{s+1}\right\rangle \oplus\left\langle x_{s+2}\right\rangle \oplus \ldots \oplus\left\langle x_{2 s}\right\rangle}{\left\langle n_{1} x_{s+1}+x_{2 s}, n_{1} x_{s+1}+n_{2} x_{s+2}, \ldots, n_{s-2} x_{2 s-2}+n_{s-1} x_{2 s-1}+x_{2 s}, 2 x_{s+2}, \ldots, 2 x_{2 s}\right\rangle} \\
& H^{3}(E, \mathbf{Z}) \cong\left\langle x_{s+1}, \ldots, x_{2 s} \mid n_{1} x_{s+1}+x_{2 s}=0, n_{1} x_{s+1}+n_{2} x_{s+2}=0, \ldots,\left(n_{s}-1\right) x_{2 s-1}=0,2 x_{s+1}=0, \ldots, 2 x_{2 s}=0\right\rangle \\
& H^{3}(E, \mathbf{Z}) \cong \underbrace{\mathbf{Z}_{2} \oplus \ldots \oplus \mathbf{Z}_{2}}_{k-1}, \text { if } k>0, \\
& \cong \mathbf{Z}_{2}, \text { if } k=0,
\end{aligned}
$$

where $k$ is the number of even $n_{i}$ 's.

$H^{4}(E, \mathbf{Z}) \cong 0$. 


\section{(ii) The Group $K$}

Here the group $K$ is given by $K=\frac{F}{R}$, where $F$ is the free group generated by $x_{1}, x_{2}$ (say), and $R$ is the normal subgroup generated by $r_{1}, r_{2}, r_{3}$ where

$$
r_{1}=x_{1}^{2}, r_{2}=x_{2}^{2 r}, r_{3}=x_{1}^{2} x_{2}^{-2}
$$

Let $\pi: \mathbf{Z F} \rightarrow \mathbf{Z} K$ be the homomorphism induced by the canonical homomorphism of $F$ onto $K$ with kennel $R$. Let $\pi\left(x_{1}\right)=h_{1}, \pi\left(x_{2}\right)=h_{2}$.

\section{Theorem 2.2}

The following is a free $\mathbf{Z} K$-resolution of $\mathbf{Z}$ :

$$
\ldots Y_{1} \stackrel{d_{3}}{\rightarrow} Y_{1} \stackrel{d_{2}}{\rightarrow} Y_{1} \stackrel{d_{3}}{\rightarrow} Y_{1} \stackrel{d_{2}}{\rightarrow} Y_{1} \stackrel{d_{1}}{\rightarrow} Y_{0} \stackrel{d_{0}}{\rightarrow} \mathbf{Z} K \stackrel{\varepsilon}{\rightarrow} \mathbf{Z} \rightarrow 0
$$

where $Y_{0}$ is a right $\mathbf{Z} K$-module free on $\alpha_{1}, \alpha_{2}$

$\mathrm{Y}_{1},, \quad, \quad, \quad, \quad, \beta_{1}, \beta_{2}, \beta_{3}$

and $\varepsilon, d_{0}, d_{1}, d_{2}, d_{3}$ are the $\mathbf{Z} K$-homomorphisms and given by

$\varepsilon(g)=1 \in \mathbf{Z}$, for all $g \in K$,

$d_{0}\left(\alpha_{i}\right)=h_{i}-1, \quad i=1,2$.

$d_{1}\left(\beta_{i}\right)=\alpha_{i}\left(h_{i}^{2 r-1}+\ldots+1\right), \quad i=1,2$.

$d_{1}\left(\beta_{3}\right)=\alpha_{1}\left(h_{1}+1\right) h_{2}^{-2}-\alpha_{2} h_{2}^{-2}\left(h_{2}+1\right)$,

$d_{2}\left(\beta_{i}\right)=\beta_{i}\left(h_{i}-1\right), i=1,2$.

$d_{2}\left(\beta_{3}\right)=-\beta_{1}+\beta_{2}+\beta_{3}\left(h_{1}^{2(r-1)}+\ldots+h_{1}^{2}+1\right)$,

$d_{3}\left(\beta_{i}\right)=\beta_{i}\left(h_{i}^{2 r-1}+\ldots+h_{i}+1\right), \quad i=1,2$.

$d_{3}\left(\beta_{3}\right)=\beta_{1}\left(h_{1}+1\right)-\beta_{2}\left(h_{2}+1\right)+\beta_{3}\left(h_{1}^{2}-1\right)$,

Let $A$ be a $Z K$-module. The homology groups $H_{n}(K, A)$ are given by the homology of the complex:

$\ldots \stackrel{\bar{d}_{3}}{\rightarrow} A^{3} \stackrel{\bar{d}_{2}}{\rightarrow} A^{3} \stackrel{\bar{d}_{3}}{\rightarrow} A^{3} \stackrel{\bar{d}_{2}}{\rightarrow} A^{3} \stackrel{\bar{d}_{1}}{\rightarrow} A^{2} \stackrel{\bar{d}_{0}}{\rightarrow} A \rightarrow 0$

where $A^{n}$ stands for the direct sum of $n$ isomorphic copies of $A$ and the homomorphisms $\bar{d}_{0}, \bar{d}_{1}, \bar{d}_{2}, \bar{d}_{3}, \bar{d}_{4}$ are induced by $d_{0}, d_{1}, d_{2}, d_{3}, d_{4}$ respectively and are given by

$\bar{d}_{0}\left(a_{1}, a_{2}\right)=\left(h_{1}-1\right) a_{1}+\left(h_{2}-1\right) a_{2}$,

$\bar{d}_{1}\left(a_{1}, a_{2}, a_{3}\right)=\left(\left(h_{1}^{2 r-1}+\ldots+1\right) a_{1}+\left(h_{1}+1\right) h_{2}^{-2} a_{3},\left(h_{2}^{2 r-1}+\ldots+1\right) a_{2}-h_{2}^{-2}\left(h_{2}+1\right) a_{3}\right)$, 
$\bar{d}_{2}\left(a_{1}, a_{2}, a_{3}\right)=\left(\left(h_{1}-1\right) a_{1}-a_{3},\left(h_{1}+1\right) h_{2}^{-2} a_{3},\left(h_{2}^{2 r-1}+\ldots+1\right) a_{2}-h_{2}^{-2}\left(h_{2}+1\right) a_{3}\right)$,

$\left.\bar{d}_{3}\left(a_{1}, a_{2}, a_{3}\right)=\left(\left(h_{1}^{2 r-1}+\ldots+1\right) a_{1}+\left(h_{1}+1\right) a_{2}+a_{3},\left(h_{2}^{2 r-1}+\ldots+1\right) a_{2}-\left(h_{2}+1\right) a_{3}\right),\left(h_{1}^{2}-1\right) a_{3}\right)$,

for some $a_{1}, a_{2}, a_{3} \in A$.

The integral homology and cohomology groups are

$H_{0}(K, \mathbf{Z}) \cong \mathbf{Z}$.

$H_{1}(K, \mathbf{Z}) \cong \mathbf{Z}_{2} \oplus \mathbf{Z}_{2 r}$.

$H_{2}(K, \mathbf{Z}) \cong 0$.

$H_{3}(K, \mathbf{Z}) \cong \mathbf{Z}_{2} \oplus \mathbf{Z}_{2 r}$.

$H_{4}(K, \mathbf{Z}) \cong 0$.

Hence $H_{2 n-1}(K, \mathbf{Z}) \cong \mathbf{Z}_{2} \oplus \mathbf{Z}_{2 r}$ and

$H_{2 n}(K, \mathbf{Z}) \cong 0$, for $n \geq 1$.

$H^{0}(K, \mathbf{Z}) \cong 0$

$H^{1}(K, \mathbf{Z}) \cong \frac{\left\{\left(a_{1}, a_{2}\right) 2 r a_{1}=0,2 r a_{2}=0, a_{1}=a_{2}\right\}}{\{(0,0)\}}$

$\cong 0$.

$H^{2}(K, \mathbf{Z}) \cong \frac{\left\{\left(a_{1}, a_{2}, a_{3}\right) \mid r a_{3}-a_{1}+a_{2}=0, a_{i} \in \mathbf{Z}\right\}}{\left\{\left(2 r a_{1}, 2 r a_{2}, 2 a_{1}-2 a_{2}\right), a_{i} \in \mathbf{Z}\right\}}$.

$\cong \frac{\left\{\left(a_{2}+r a_{3}, a_{2}, a_{3}\right) \mid a_{i} \in \mathbf{Z}\right\}}{\left\{\left(2 r a_{1}, 2 r a_{2}, 2 a_{1}-2 a_{2}\right) \mid a_{i} \in \mathbf{Z}\right\}}$.

$\cong \frac{\langle(r, 0,1)\rangle \oplus\langle(1,1,0)\rangle}{\langle 2(r, 0,1)+2(o, r,-1)\rangle}$

$\cong \frac{\langle x\rangle \oplus\langle y\rangle}{\langle 2 y, 2(r x-y)\rangle}$,

writing $x=(1,1,0)$ and $y=(r, 0,1)$.

$\cong \mathbf{Z}_{2} \oplus \mathbf{Z}_{2 r}$

$H^{3}(K, \mathbf{Z}) \cong \frac{\left\{\left(a_{1}, a_{2}, a_{3}\right) \mid 2 r a_{1}=0=2 r a_{2}, a_{1}=a_{2} ; a_{i} \in \mathbf{Z}\right\}}{\left\{\left(0,0, r a_{3}-a_{1}+a_{2}\right) ; a_{i} \in \mathbf{Z}\right\}}$

$\cong \frac{\left\{\left(0,0, a_{3}\right) ; a_{3} \in \mathbf{Z}\right\}}{\left\{\left(0,0, r a_{3}-a_{1}+a_{2}\right) ; a_{i} \in \mathbf{Z}\right\}}$ 


$$
\begin{aligned}
& \cong \frac{\langle(0,0,1)\rangle}{\langle(0,0, r),(0,0,-1),(0,0,1)\rangle} \\
& \cong\langle x \mid r x=0,-x=0, x=0\rangle \\
& \cong 0, \\
& H^{4}(K, \mathbf{Z}) \cong \frac{\left\{\left(a_{1}, a_{2}, a_{3}\right) \mid r a_{3}-a_{1}+a_{2}=0, a_{i} \in \mathbf{Z}\right\}}{\left\{\left(2 r a_{1}, 2 r a_{2}, 2 a_{1}-2 a_{2}\right)\right\}} . \\
& \cong H^{2}(K, \mathbf{Z}) \cong \mathbf{Z},
\end{aligned}
$$

Hence $H^{2 n+1}(K, \mathbf{Z}) \cong\{0\}, \quad n \geq 0$,

$$
H^{2 n}(K, \mathbf{Z}) \cong \mathbf{Z}_{2} \oplus \mathbf{Z}_{2 r}, \quad n \geq 1 .
$$

(iii) The Group $G$ of Hyperbolic Isometries with Infinite Generators and Relations

The group $G$ is given by $G=\frac{F}{R}$, where $F$ is the free group generated by, say, $x_{1}, x_{2}, x_{3}, \ldots$ and $R$ is the normal subgroup of $F$ generated by $r_{1}, r_{2}, r_{3}, \ldots$ where

$$
r_{2 k-1}=x_{k}^{2}, r_{2 k}=\left(x_{k} x_{k+1}\right)^{n_{k}}, \quad k=1,2,3, \ldots
$$

Although the number of generators and relation of $G$ are infinite, our method of construction of the free resolution as described in [18] is still valid for $G$, since (i) each generator of the group occurs only in the finite number of relations, (ii) each arbitrary group-ring element corresponding to a free generator of the solution module occurs as a co-efficient in the value of a finite number of unknowns at each stage of solving the set of the relevant linear equations. Here we have obtained a 6-term partial resolution which yields the corresponding homology immediately. However the cohomology can't be determined from this resolution since the free module of the resolution are infinitely generated, and in general,

$\operatorname{Hom}\left(\sum_{i \in l} A_{i}, B\right)$ is not isomorphic to $\sum_{i \in l} \operatorname{Hom}\left(A_{i}, B\right)$, when I is infinite.

Let $\pi: \mathbf{Z} F \rightarrow \mathbf{Z} G$ be the homomorphism induced by the canonical homomorphism of $F$ onto $G$ with $R$ as the kernel, let $\pi\left(x_{i}\right)=h_{i}$.

\section{Theorem 2.3}

The following is a free 6-term partial $\mathbf{Z} G$-resolution of $\mathbf{Z}$ :

$$
Y_{4} \stackrel{d_{4}}{\rightarrow} Y_{3} \stackrel{d_{3}}{\rightarrow} Y_{2} \stackrel{d_{2}}{\rightarrow} Y_{1} \stackrel{d_{1}}{\rightarrow} Y_{0} \stackrel{d_{0}}{\rightarrow} \mathbf{Z} G \stackrel{\varepsilon}{\rightarrow} \mathbf{Z} \rightarrow 0
$$

where $Y_{0}$ is a right $\mathbf{Z} G$-module free on $\alpha_{1}, \alpha_{2}, \alpha_{3}, \ldots$

$$
Y_{1}, \quad, \quad, \quad, \quad, \beta_{1}, \beta_{2}, \beta_{3}, \ldots
$$




$$
\begin{array}{ccccc}
Y_{2}, & , & , & , & , \delta_{1}, \delta_{2}, \delta_{3}, \ldots \\
Y_{3}, & , & , & , & , \lambda_{1}, \lambda_{2}, \lambda_{3}, \ldots \\
Y_{4}, & , & , & , & , \mu_{1}, \mu_{2}, \mu_{3}, \ldots
\end{array}
$$

and $\varepsilon, d_{0}, d_{1}, d_{2}, d_{3}, d_{4}$ are the $\mathbf{Z} G$-homomorphisms and given by

$\varepsilon(g)=0$, for all $g \in G$,

$d_{0}\left(\alpha_{i}\right)=h_{i}-1, \quad i=1,2,3, \ldots$.

$d_{1}\left(\beta_{2 i-1}\right)=\alpha_{i}\left(h_{i}+1\right)$ and

$d_{1}\left(\beta_{2 i}\right)=\alpha_{i} h_{i+1}\left[\left(h_{i} h_{i+1}\right)^{n_{i 1}-1}+\ldots+1\right]+\alpha_{i+1}\left[\left(h_{i} h_{i+1}\right)^{n_{i}-1}+\ldots+1\right], \quad i=1,2,3, \ldots$.

$d_{2}\left(\delta_{4 i-3}\right)=\beta_{2 i-1}\left(h_{i}-1\right)$,

$d_{2}\left(\delta_{2 i}\right)=\beta_{2 i}\left(h_{i} h_{i+1}-1\right)$ and

$d_{2}\left(\delta_{4 i-1}\right)=-\left(\beta_{2 i-1}+\beta_{2 i+1}\right)\left[\left(h_{i} h_{i+1}\right)^{n_{i}-1}+\ldots+1\right]+\beta_{2 i}\left(h_{i+1}+1\right)$, for $i=1,2,3, \ldots$.

$d_{3}\left(\lambda_{8 i-7}\right)=\delta_{4 i-3}\left(h_{i}+1\right)$,

$d_{3}\left(\lambda_{2 i}\right)=\delta_{4 i-1}\left(h_{i} h_{i+1}-1\right)-\delta_{2 i}\left[\left(h_{i} h_{i+1}\right)^{n_{i}-2}+\ldots+1\right]\left(h_{i+1}+1\right)$,

$d_{3}\left(\lambda_{4 i-1}\right)=\delta_{2 i}\left[\left(h_{i} h_{i+1}\right)^{n_{i}-1}+\ldots+1\right]$ and

$d_{3}\left(\lambda_{8 i-3}\right)=\left(\delta_{4 i-3}+\delta_{4 i+1}\right)\left[\left(h_{i} h_{i+1}\right)^{n_{i}-1}+\ldots+1\right]+\delta_{4 i-1}\left(h_{i+1}-1\right)$, for $i=1,2,3, \ldots$.

$d_{4}\left(\mu_{16 i-15}\right)=\lambda_{8 i-7}\left(h_{i}-1\right)$,

$d_{4}\left(\mu_{2 i}\right)=\lambda_{8 i-3}\left(h_{i} h_{i+1}-1\right)-\lambda_{2 i}\left[\left(h_{i} h_{i+1}\right)^{n_{i}-2}+\ldots+1\right]\left(h_{i+1}+1\right)$,

$d_{4}\left(\mu_{4 i-1}\right)=\lambda_{2 i}\left[\left(h_{i} h_{i+1}\right)^{n_{i}-1}+\ldots+1\right]+\lambda_{2 i-1}\left[\left(h_{i} h_{i+1}\right)^{n_{i}-2}+\ldots+1\right]\left(h_{i+1}+1\right)$,

$d_{4}\left(\mu_{8 i-3}\right)=\lambda_{4 i-1}\left(h_{i} h_{i+1}-1\right)$ and

$d_{4}\left(\mu_{16 i-7}\right)=\lambda_{8 i-3}\left(h_{i+1}+1\right)-\left(\lambda_{8 i-7}+\lambda_{8 i-1}\right)\left[\left(h_{i} h_{i}\right)^{n_{i}-1}+\ldots+1\right]$, for $i=1,2,3, \ldots$.

\section{Homology Groups of $G$}

Let $A$ be a left $\mathbf{Z} G$-module. The homology groups $H_{n}(G, A)$ are given by the homology of the complex:

$$
A^{\infty} \stackrel{\bar{d}_{4}}{\rightarrow} A^{\infty} \stackrel{\bar{d}_{3}}{\rightarrow} A^{\infty} \stackrel{\bar{d}_{2}}{\rightarrow} A^{\infty} \stackrel{\bar{d}_{1}}{\rightarrow} A^{\infty} \stackrel{\bar{d}_{0}}{\rightarrow} A^{\infty} \rightarrow 0
$$

where $A^{\infty}$ stands for the direct sum of countably infinite copies of $\mathrm{A}$ and $\bar{d}_{i}$ are induced by $d_{i}$, for $i=0,1,2,3,4$ and are given by

$$
\bar{d}_{0}\left(a_{1}, a_{2}, a_{3}, \ldots\right)=\left(h_{1}-1\right) a_{1}+\left(h_{2}-1\right) a_{2}+\left(h_{3}-1\right) a_{3}+\ldots,
$$




$$
\begin{aligned}
& \bar{d}_{1}\left(a_{1}, a_{2}, a_{3}, \ldots\right)=\left(\left(h_{1}+1\right) a_{1}+h_{2}\left[\left(h_{1} h_{2}\right)^{n_{1}-1}+\ldots+1\right] a_{2},\left[\left(h_{1} h_{2}\right)^{n_{1}-1}+\ldots+1\right] a_{2}\right. \\
& +\left(h_{2}+1\right) a_{3}+h_{3}\left[\left(h_{2} h_{3}\right)^{n_{2}-1}+\ldots+1\right] a_{4},\left[\left(h_{2} h_{3}\right)^{n_{2}-1}+\ldots+1\right] a_{4}+ \\
& \left.\left(h_{3}+1\right) a_{5}+h_{4}\left[\left(h_{3} h_{4}\right)^{n_{3}-1}+\ldots+1\right] a_{6}, \ldots\right), \\
& \bar{d}_{2}\left(a_{1}, a_{2}, a_{3}, \ldots\right)=\left(\left(h_{1}-1\right) a_{1}-\left[\left(h_{1} h_{2}\right)^{n_{1}-1}+\ldots+1\right] a_{3},\left(h_{2}+1\right) a_{3}+\left(h_{1} h_{2}-1\right) a_{2},\right. \\
& -\left[\left(h_{1} h_{2}\right)^{n_{1}-1}+\ldots+1\right] a_{3}-\left[\left(h_{2} h_{3}\right)^{n_{2}-1}+\ldots+1\right] a_{7}+\left(h_{2}-1\right) a_{5}, \\
& \left(h_{2} h_{3}-1\right) a_{4}+\left(h_{3}+1\right) a_{7},\left(h_{3}-1\right) a_{9}-\left[\left(h_{2} h_{3}\right)^{n_{2}-1}+\ldots+1\right] a_{7}- \\
& \left.\left[\left(h_{3} h_{4}\right)^{n_{3}-1}+\ldots+1\right] a_{11}, \ldots\right), \\
& \bar{d}_{3}\left(a_{1}, a_{2}, a_{3}, a_{4}, \ldots\right)=\left(\left(h_{1}+1\right) a_{1}+\left[\left(h_{1} h_{2}\right)^{n_{1}-1}+\ldots+1\right] a_{5},-\left[\left(h_{1} h_{2}\right)^{n_{1}-2}+\ldots+1\right]\left(h_{2}+1\right) a_{2}+\right. \\
& {\left[\left(h_{1} h_{2}\right)^{n_{1}-1}+\ldots+1\right] a_{3},\left(h_{1} h_{2}-1\right) a_{2}+\left(h_{2}-1\right) a_{5},} \\
& {\left[\left(h_{2} h_{3}\right)^{n_{2}-1}+\ldots+1\right]\left(h_{3}+1\right) a_{4}+\left[\left(h_{2} h_{3}\right)^{n_{2}-1}+\ldots+1\right] a_{7},} \\
& {\left[\left(h_{2} h_{3}\right)^{n_{2}-2}+\ldots+1\right] a_{13}+\left[\left(h_{1} h_{2}\right)^{n_{1}-1}+\ldots+1\right] a_{5}+\left(h_{2}+1\right) a_{9},-} \\
& \left.\left[\left(h_{3} h_{4}\right)^{n_{3}-2}+\ldots+1\right]\left(h_{4}+1\right) a_{6}+\left[\left(h_{3} h_{4}\right)^{n_{3}-1}+\ldots+1\right] a_{11},\left(h_{2} h_{3}-1\right) a_{4}+\left(h_{3}-1\right) a_{13}, \ldots\right) \\
& \bar{d}_{4}\left(a_{1}, a_{2}, a_{3}, a_{4}, \ldots\right)=\left(\left(h_{1}-1\right) a_{1}-\left[\left(h_{1} h_{2}\right)^{n_{1}-1}+\ldots+1\right] a_{9},-\left[\left(h_{1} h_{2}\right)^{n_{1}-2}+\ldots+1\right]\left(h_{2}-1\right) a_{2}\right. \\
& +\left[\left(h_{1} h_{2}\right)^{n_{1}-1}+\ldots+1\right] a_{3},\left[\left(h_{1} h_{2}\right)^{n_{1}-2}+\ldots+1\right]\left(h_{2}+1\right) a_{3}+\left(h_{1} h_{2}-1\right) a_{5},- \\
& {\left[\left(h_{2} h_{3}\right)^{n_{2}-2}+\ldots+1\right]\left(h_{3}-1\right) a_{4}+\left[\left(h_{2} h_{3}\right)^{n_{2}-1}+\ldots+1\right] a_{7},\left(h_{1} h_{2}-1\right) a_{2}+} \\
& \left(h_{2}+1\right) a_{9},-\left[\left(h_{3} h_{4}\right)^{n_{3}-2}+\ldots+1\right]\left(h_{4}-1\right) a_{6}+\left[\left(h_{3} h_{4}\right)^{n_{3}-1}+\ldots+1\right] a_{11}, \\
& {\left[\left(h_{2} h_{3}\right)^{n_{2}-2}+\ldots+1\right]\left(h_{3}+1\right) a_{7}+\left(h_{2} h_{3}-1\right) a_{13},-\left[\left(h_{4} h_{5}\right)^{n_{4}-2}+\ldots+1\right]\left(h_{5}-1\right) a_{8}+} \\
& \left.\left[\left(h_{4} h_{5}\right)^{n_{4}-1}+\ldots+1\right] a_{15},\left(h_{2}-1\right) a_{17}+\left[\left(h_{1} h_{2}\right)^{n_{1}-1}+\ldots+1\right) a_{9}-\left[\left(h_{2} h_{3}\right)^{n_{2}-1}+\ldots+1\right] a_{25}, \ldots\right),
\end{aligned}
$$

for all $a_{i} \in A, i=1,2,3,4, \ldots$.

The integral homology groups of $G$ are

$H_{0}(G, \mathbf{Z}) \cong \mathbf{Z}$,

$$
\begin{aligned}
H_{1}(G, \mathbf{Z}) & \cong \frac{\left\{\left(a_{1}, a_{2}, a_{3}, a_{4}, \ldots\right) \mid a_{i} \in \mathbf{Z}\right\}}{\left\{\left(2 a_{1}+n_{1} a_{2}, n_{1} a_{2}+2 a_{3}+n_{2} a_{4}+2 a_{5}+n_{3} a_{6}, \ldots\right) \mid a_{i} \in \mathbf{Z}\right\}} \\
& \cong \frac{\left\{a_{1}(1,0,0, \ldots)+a_{2}(0,1,0, \ldots)+a_{3}(0,0,1,0, \ldots)+a_{4}(0,0,0,1,0, \ldots), \ldots \mid a_{i} \in \mathbf{Z}\right\}}{\left\{a_{1}(2,0,0, \ldots)+a_{2}\left(n_{1}, n_{1}, 0, \ldots\right)+a_{3}(0,2,0, \ldots)+a_{4}\left(0, n_{2}, n_{2}, 0, \ldots\right)+a_{5}(0,0,2,0, \ldots)\right\}} \\
& \cong \frac{\left\langle x_{1}\right\rangle \oplus\left\langle x_{2}\right\rangle \oplus\left\langle x_{3}\right\rangle \ldots}{\left\langle 2 x_{1}, n_{1}\left(x_{1}+x_{2}\right), 2 x_{2}, n_{2}\left(x_{2}+x_{3}\right), 2 x_{3}, \ldots\right\rangle},
\end{aligned}
$$


writing $x_{i}=(0, \ldots, 0,1,0,0, \ldots)$, where 1 is in the $i$-th position,

$$
\cong\left\langle x_{1}, x_{2}, x_{3}, x_{4}, \ldots \mid 2 x_{1}=0,2 x_{2}=0,2 x_{3}=0, \ldots, n_{1}\left(x_{1}+x_{2}\right)=0, n_{2}\left(x_{2}+x_{3}\right)=0, \ldots\right\rangle
$$

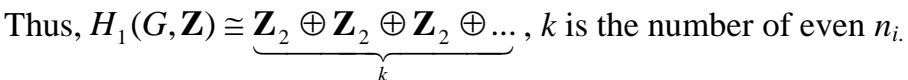

$H_{2}(G, \mathbf{Z}) \cong 0$.

$$
\begin{aligned}
H_{3}(G, \mathbf{Z}) & \cong \frac{\left\{\left(a_{1}, a_{2}, a_{3}, a_{4}, \ldots\right) \mid-n_{1} a_{3}=0,2 a_{3}=-n_{1} a_{3}-n_{2} a_{7}=2 a_{7}=0=-n_{2} a_{7}-n_{3} a_{11} ; a_{i} \in \mathbf{Z}\right\}}{\left\{\left(2 a_{1}+n_{1} a_{5},-2\left(n_{1}-1\right) a_{2}+n_{1} a_{3}, 0,-2\left(n_{2}-1\right) a_{4}+n_{2} a_{7}, \ldots\right) \mid a_{i} \in \mathbf{Z}\right\}} \\
& \cong \frac{\left\langle x_{1}\right\rangle \oplus\left\langle x_{2}\right\rangle \oplus\left\langle x_{3}\right\rangle \oplus\left\langle x_{4}\right\rangle \oplus \ldots}{\left\langle 2 x_{1}, n_{1} x_{1}, 2\left(n_{1}-1\right) x_{2}, n_{1} x_{2}, 2 x_{2}, 2\left(n_{2}-1\right) x_{3}, n_{2} x_{3}, \ldots\right\rangle}
\end{aligned}
$$

where $x_{i}=(0, \ldots, 0,1,0, \ldots, 0)$ with 1 is an the $i$-th position,

$$
\cong\left\langle x_{1}, x_{2}, x_{3}, x_{4}, \ldots \mid 2 x_{1}=0, n_{1} x_{1}=0,2\left(n_{1}-1\right) x_{2}=0, n_{1} x_{2}=0,2\left(n_{2}-1\right) x_{3}=0, n_{2} x_{3}=0 \ldots\right\rangle .
$$

Hence $H_{3}(G, \mathbf{Z}) \cong Z_{2} \oplus \sum_{i} \mathbf{Z}_{h_{i+1}}$ or $\sum_{i} \mathbf{Z}_{h_{i+1}}$,

where $i$ runs over all those integers $i \geq 2$ for which $n_{i}$ is even, and $h_{i+1}$ is the h.c.f. of $2\left(n_{i}-1\right)$ and $n_{i}$, according as $n_{i}$ is even or odd

$H_{4}(G, \mathbf{Z}) \cong 0$.

\section{REFERENCES}

[1] Akhter, N. and Majumdar, S., Determination of the homology and the cohomology of NEC group with non-orientable orbit spaces and no reflexions, Bull. Call. Math. Soc., 95(3), (2003), p. 207-218.

[2] Huebschmann, J., Perturbation theory and free resolutions for nilpotent groups of class 2, J. Algebra, 126, (1989), 348-399.

[3] Huebschmann, J., Cohomology of nilpotent groups of class 2, J. of Algebra, 126, (1989), 400-450.

[4] Huebschmann J., The mod $p$ cohomology rings of metacyclic group, J. Pure Appl. Algebra, 60, (1989), 53-103.

[5] Huebschmann, J., Cohomological theory of aspherical groups and of small cancellation groups, J. Pure Appl. Algebra, 14, (1993), 137-143.

[6] Learner, A., Cohomology groups, Lecturer notes, Queen Mary College, University of London,(1965).

[7] Lyndon, R.C., Cohomology theory of groups with a single defining relation, Ann. Math. 52, 3, (1950),656-665.

[8] Macbeath A. and Hoare, A. H. M., Groups of hyperbolic crystallography, J. Math. Proc. Cambridge Philos. Soc. 79, (1976), 235-249.

[9] MacLane, S., Homology, Springer-Verlag, Berlin, 1963.

[10] Majumdar, S., A free resolution for a class of groups, J. Lond. Math. Soc., 2, 2, (1970), 615-619.

[11] Majumdar, S., A free resolution for a general class of groups, Tech. report Math. stat. (17), 17, (1971), ISI, Calcutta.

[12] Majumdar, S., Free resolution for certain classes of groups, Proc. Edin. Math. Soc., 26, (1983), 173179. 
[13] Majumdar, S., Homology and cohomology of a class of polycyclic groups, Internal report, IC/84/177, ICTP, (1984), Trieste, Italy.

[14] Majumdar, S., On the homology and cohomology of certain polycyclic groups, Internal report, IC/87/305, ICTP, (1987), Trieste, Italy.

[15] Majumdar, S. and Akhter, N., Determination of the homology and the cohomolgy of the trifoil knot group and the fundamental group of an interesting manifold, Ganit (J. Bangladesh, Math. Soc.,) 22, (2002), 7-14.

[16] Majumdar, S. and Akther, N., Computation of homology and cohomology groups of a metacyclic group and a factor group of the Heisenberg group, Ganit (J. Bangladesh Math. Soc.) 31, (2011) 9-22.

[17] Majumdar, S. and Akhter N., Determination of the homology and the cohomolgy of a class of infinite groups associated with groups of Fibonacci type, Bull. Call. Math. Soc., 100, 2008.

[18] Majumdar, S. and Akhter N., On a simple method of determining the homology and the cohomology of finitely presented groups, J. of Bangladesh Academy of Sciences, 34(2), (2010), 103-114.

[19] McCullough, D., Miller, A. and Zimmermann, B., Group action on nonclosed 2-manifolds, J. of Pure and Applied Algebra, 64, (1990), 269-292. 